\title{
IMPLANTAÇÃO DE UM SERVIÇO DE CIRURGIA AMBULATORIAL: O PAPEL DA ENFERMAGEM NESSE CENÁRIO
}

\author{
Maria Cristina Simões Flório* \\ Cristina Maria Galvão**
}

FLÓRIO, M.C.S.; GALVÃO, C.M. Implantação de um serviço de cirurgia ambulatorial: o papel da enfermagem nesse cenário. Rev. latino-am.enfermagem, Ribeirão Preto, v. 6, n. 5, p. 83-88, dezembro 1998.

O presente estudo tem como objetivo apresentar a trajetória percorrida pelo enfermeiro na implantação de um serviço de cirurgia ambulatorial em um hospital geral. Aborda a importância deste serviço no contexto atual, discorre sobre as atividades desenvolvidas pelo enfermeiro e equipe de enfermagem, bem como os desafios e conquistas enfrentados no dia a dia de trabalho.

UNITERMOS: cirurgia ambulatorial, atividades do enfermeiro, equipe de enfermagem

De acordo com WATSON \& SANGERMANO ${ }^{16}$, nos Estados Unidos da América (EUA) em 1818, surgiu no Massachusetts General Hospital o primeiro departamento para pacientes externos; entretanto, as autoras salientam que somente na década de 60 a comunidade médica passou a interessar-se pela cirurgia ambulatorial, devido aos avanços nas áreas anestésica e cirúrgica, os quais possibilitaram uma recuperação ágil e com menor número de complicações do paciente cirúrgico.

Segundo Ford apud DAVIS ${ }^{6}$ no início dos anos 70, foi inaugurado o Surgicenter em Phoenix (EUA), pelos anestesiologistas Dr. Wallace Reed e Dr. John Ford que definiram esta unidade como destinada " a proporcionar assistência cirúrgica qualificada ao paciente cuja operação é por demais complexa para o consultório, porém sem a proporção que exija hospitalização". MATHIAS ${ }^{10}$ aborda que neste serviço foram estabelecidos pelos fundadores, muitos conceitos e práticas que influenciam até hoje o campo da cirurgia ambulatorial, tais como: critérios de seleção de pacientes, tipos de procedimentos anestésicos e técnicas cirúrgicas mais apropriados, bem como conceitos relativos a alta e acompanhamento do paciente.

Para Cohen apud DAVIS ${ }^{6}$, a cirurgia ambulatorial tem como premissa, aquela operação onde o paciente chegará ao hospital, realizará o procedimento com a maior segurança possível e retornará para seu ambiente familiar o mais rápido possível, não colocando sua integridade física em risco. Quando necessário este terá sua internação providenciada, isso raramente ocorrerá, pois o paciente deverá ser avaliado previamente pelo cirurgião.

A organização de um serviço ambulatorial pode ser realizada de três formas, segundo OLIVEIRA FILHO $^{12}$ :

- Unidade Ambulatorial Independente: quando localiza-se anexa ao hospital usufruindo das estruturas deste (radiologia, laboratório). As estruturas de secretaria, centro cirúrgico e recuperação são independentes dos pacientes internados.

- Unidade Ambulatorial Integrada à Atividade Interna do Hospital: é desenvolvida em hospitais já estruturados, sem grandes áreas físicas disponíveis, mas com um centro cirúrgico ocioso, no qual algumas salas são destinadas ao atendimento ambulatorial.

- Unidade Ambulatorial Autônoma: são atividades desenvolvidas em serviços bem estruturados, mas sem leito para internação.

Segundo YAMASHITA ${ }^{17}$, as vantagens do serviço de cirurgia ambulatorial são: diminuição do número de internações, tendo assim um custo hospitalar reduzido em 25\% a 75\%; liberação de leitos hospitalares; redução da ansiedade e oferecimento de maior conforto aos pacientes e acompanhantes; permite retorno precoce ao lar e ao trabalho, além de diminuir o risco de infecção hospitalar.

Aproximadamente dez anos trabalhando em áreas

\footnotetext{
* Enfermeira. Supervisora de Centro Cirúrgico da Fundação Waldemar B.Pessoa., Ribeirão Preto-SP

** Enfermeira. Professora Doutora junto ao Departamento de Enfermagem Geral e Especializada da Escola de Enfermagem de Ribeirão Preto, Universidade de São Paulo
} 
cirúrgicas, a princípio como auxiliar de enfermagem em um hospital voltado para o atendimento do sexo feminino, desenvolvendo atividades nos setores de recuperação e centro cirúrgico, acarretou um despertar em relação à assistência de enfermagem prestada ao paciente submetido ao ato anestésico - cirúrgico. Essa situação motivou a procura de conhecimento e preparo afim de atuar no campo, inicialmente pensávamos na área de anestesia. Após a formação ao nível superior, a oportunidade não surgiu e com isso fomos trabalhar em um hospital geral, onde desenvolvemos atividades em diferentes unidades hospitalares. Na nossa trajetória profissional sempre houve identificação com as áreas chamadas fechadas; principalmente onde havia pacientes pós-operados.

No ano de 1994, fomos convidadas a assumir o setor que estava sendo criado com a denominação de pequena cirurgia, onde inicialmente seriam atendidos pacientes que necessitassem de procedimentos como biópsias, alguns tipos de suturas e operações que não pudessem ser realizadas em consultórios.

Inicialmente, a direção do hospital pensou criar um serviço que oferecesse maior agilidade para os cirurgiões e os pacientes. Com o decorrer do tempo percebeu-se que a estrutura montada poderia abrigar procedimentos mais complexos. Foi realizada uma listagem junto às equipes médicas para que indicassem os procedimentos a serem realizados ao nível ambulatorial.

Após o levantamento foi montado o serviço para que houvesse redução na demanda do centro cirúrgico principal; diminuição do número de internações desnecessárias, reduzindo assim, custos, risco de infecções hospitalares; acarretasse agilidade no atendimento e aumento do número de cirurgias no hospital como um todo.

O hospital, no qual o serviço acima descrito foi montado é do tipo geral com um número, em torno de mil e cem pacientes cirúrgicos por mês, onde são realizadas cirurgias cardíacas, neurológicas, ortopédicas, transplantes renais, dentre outras e presta serviços há cinqüenta e dois anos; atualmente tornou-se totalmente privado criando seu próprio plano de saúde.

O serviço de cirurgia ambulatorial foi criado com a denominação de "Hospital Dia", para realização de cirurgia onde o paciente pudesse retornar para casa no mesmo dia.

Nos EUA, conforme já mencionamos, a cirurgia ambulatorial tornou-se uma realidade a partir da década de 60 e desde então, ocorreu um aumento acentuado deste tipo de serviço no país. De acordo com LLEWELLYN ${ }^{9}$ esta tendência persistirá, devido às inovações tecnológicas, o aumento de satisfação do cliente, bem como políticas governamentais e de grupos privados voltadas para a redução de custos.
Para NOCITE ${ }^{11}$, no Brasil, existe uma tendência de expansão do número de procedimentos ambulatoriais, principalmente devido a necessidade de redução de custos, maior disponibilidade de leitos hospitalares, diminuição dos aspectos negativos referentes a internação, tais como: separação do convívio familiar e infecção cruzada.

Ao nos transportarmos para o cenário da enfermagem brasileira, existem alguns estudos como os de COLOGNA et al. ${ }^{3}$; GUIDO $^{8}$; CASTRO et al. ${ }^{2}$; CÁRNIO et al. ${ }^{1}$, que abordam diferentes aspectos sobre cirurgia ambulatorial, entretanto, esta temática ainda é bastante inexplorada em nosso meio. Assim, procurando oferecer nossa contribuição, o presente estudo tem como objetivo apresentar a trajetória percorrida pelo enfermeiro na implantação de um serviço de cirurgia ambulatorial em um hospital geral.

\section{CARACTERIZAÇÃO DO SERVIÇO DE CIRURGIA AMBULATORIAL}

Inicialmente, as pessoas envolvidas na montagem do serviço em questão eram: enfermeiro, médicos (anestesista e cirurgião), gerente administrativo e o diretor clínico do hospital. Nossa estrutura física foi montada num local que deveria abrigar uma unidade de terapia intensiva, contando com toda infra-estrutura necessária.

A princípio o serviço funcionou com duas salas cirúrgicas, atendendo pacientes do plano de saúde do hospital e realizou-se pequenos procedimentos (biópsias, lesão de pele, etc.), chegando a um total de oitenta a cem pacientes nos primeiros meses.

Com o sucesso e passado o período de experiência, a infra-estrutura foi adequada para a realização de cirurgias de médio porte (nódulo de mama, hérnias umbilicais e inguinais, etc.), implantamos ainda, procedimentos com outros tipos de anestesia que não só local, como: geral, peridural, intercostal etc. Neste período surgiram algumas dificuldades como a recuperação pósanestésica dos pacientes; necessidade de treinamento dos membros da equipe de enfermagem que não possuíam experiência com paciente cirúrgico; além de problemas como falta de material de consumo e instrumental; número reduzido de pessoal; uniforme privativo adequado para adentrar no bloco cirúrgico. Após um tempo aproximado de dois meses percebemos que a idéia do serviço de cirurgia ambulatorial era bem aceita pela equipe médica e clientela atendida e a ampliação do local foi realizada.

Primeiramente, a planta física foi adequada passando a funcionar com três salas cirúrgicas, recuperação pós-anestésica com quatro leitos, recepção dos pacientes pré-cirúrgicos e acompanhantes, vestiários individuais para equipe médica (feminino e masculino), 
vestiário para pacientes, arsenal de medicamentos, sala de recuperação tardia (onde o paciente aguarda em cadeira reclinável a alta hospitalar).

O objetivo do serviço é a realização do procedimento cirúrgico de pequeno e médio porte ao nível ambulatorial, sem o comprometimento da integridade dos clientes e com base na regulamentação do Conselho Federal de Medicina ${ }^{4}$, o qual determina que o ambiente tem que contemplar estrutura em condições higiênicas e sanitárias; esterilização e desinfecção dos instrumentos; ter registro de todos os procedimentos realizados; condições para práticas anestésicas; garantia de suporte hospitalar em eventuais casos de internação; garantia de assistência no pós alta, em decorrência de complicações durante vinte e quatro horas. No serviço em questão, este suporte é realizado pelo pronto atendimento da unidade de emergência.

Para a redução de intercorrências no serviço, alguns critérios de seleção do paciente para o tratamento cirúrgico ambulatorial foram estabelecidos, sendo eles: o paciente não apresentar comprometimento sistêmico grave, seja por doenças ou pela doença cirúrgica; pacientes com distúrbios sistêmicos moderados ou doenças gerais compensadas e o procedimento não necessitar de cuidados especiais no pós-operatório deverão estar acompanhados de um adulto, lúcido e previamente identificado; as cirurgias devem ser realizadas preferencialmente até às quatorze horas (para o paciente ter condições de recuperação e alta hospitalar até às vinte horas); a residência do paciente não deve exceder a distância máxima de cinqüenta quilômetros do serviço; o acompanhante terá que estar presente nas orientações pós-operatórias, bem como na determinação da unidade de atendimento em eventuais intercorrências; os procedimentos anestésicos-cirúrgicos não devem ultrapassar um período de duas horas; as cirurgias deverão ser agendadas pelo cirurgião, onde o mesmo deverá definir a presença ou não do anestesista.

No primeiro horário são marcadas três cirurgias com a presença do anestesista e outras duas com anestesia local num total de cinco salas; não havendo preferência de equipes. O horário de atendimento é das sete às vinte horas e deverá respeitar as seguintes determinações: com presença de anestesista até às quatorze horas e com anestesia local até às dezessete horas.

Em relação ao processo de admissão do paciente, o mesmo chega ao serviço mediante a orientação do cirurgião, que já agendou sua cirurgia previamente, no mínimo com quinze dias de antecedência. Cabe ao cirurgião solicitar todos os exames pré-operatórios e outros tipos de avaliações, por exemplo cardiológica. Como o paciente pertence ao plano de saúde do hospital, ele recebe um impresso no ato da autorização da cirurgia, o qual contém orientações gerais para o seu preparo, tais como: horário para fazer tricotomia e local; horário de chegada no hospital; manter jejum de oito horas quando a cirurgia não for com anestesia local, dentre outras.

Ao chegar no serviço o paciente é recepcionado e a pessoa que realiza sua admissão avisa o bloco cirúrgico ambulatorial, bem como o anestesista que fará avaliação pré-anestésica. Após avaliação o paciente é encaminhado ao vestiário, onde coloca roupas do hospital. A seguir, é levado para a sala cirúrgica onde a equipe o aguarda. Ao término do ato anestésico cirúrgico o paciente é encaminhado para a recuperação, onde permanece até sua alta hospitalar.

A alta hospitalar dependerá de alguns critérios definidos pelo serviço a saber: o paciente apresenta quadro de estabilidade dos sinais vitais pelo período de uma hora; ausência de náuseas e vômitos e também de dificuldade respiratória; capacidade de ingerir líquidos; condições de locomoção como antes da realização do procedimento, presença mínima de sangramento e dor; ausência de retenção urinária; estar orientado no tempo e espaço; ter um acompanhante adulto.

\section{AS ATIVIDADES DA ENFERMAGEM NO SERVICCO DE CIRURGIA AMBULATORIAL}

O enfermeiro do serviço de cirurgia ambulatorial é o responsável pela estrutura organizacional e dinâmica de funcionamento desta unidade. Acreditamos que compete a este profissional o gerenciamento da assistência de enfermagem prestada ao paciente, sendo sua ação direcionada para o desenvolvimento de atividades administrativas, assistenciais, educativas e de pesquisa com vistas ao aprimoramento da prática.

Desta forma, fundamentadas nos estudos de PRAÇA \& AZEVEDO ${ }^{13}$; ROZA $^{14}$; SILVA et al. ${ }^{15}$, passaremos a listar as principais atividades desenvolvidas pelo enfermeiro do serviço de cirurgia ambulatorial em questão. Em relação as atividades assistenciais, destacamos:

- recepção do paciente na sala cirúrgica, checando dados referentes a identificação e preparo pré-operatório;

- atendimentos das solicitações emanadas do paciente durante toda sua permanência no serviço;

- avaliação física e emocional do paciente na sala de recuperação pós-anestésica;

- realização de orientações relativas aos cuidados necessários no pós-operatório para o paciente e familiares. $\mathrm{O}$ enfermeiro do serviço de cirurgia ambulatorial realiza diversas atividades administrativas, tais como:

- elaboração da programação cirúrgica diária e sua distribuição em cada sala; 
- realização de escalas (diária, mensal e férias) do pessoal de enfermagem;

- participação em diferentes reuniões para organização dos serviços do hospital como um todo (Comissão de Controle de Infecção Hospitalar e Comissão de Controle da Qualidade de Produtos);

- realização de relatórios mensais e anuais de procedimentos anestésicos-cirúrgicos, equipes médicas, ocupação por sala, convênios atendidos;

- previsão e controle semanal de materiais provenientes do almoxarifado e farmácia;

- revisão dos gastos de sala e encaminhamento para o setor financeiro;

- controle da freqüência, falta e atrasos do pessoal de enfermagem;

- supervisão do funcionamento de todas as salas de cirurgias;

- orientação e supervisão da organização e limpeza da unidade;

- delegação e supervisão da montagem das sala de cirurgias;

- atendimento das solicitações emanadas das equipes médicas e pessoal de enfermagem;

- realização de reuniões com as equipes médicas, para implementação de estratégias com vistas a melhoria do serviço;

- realização de reuniões com o pessoal de enfermagem, para resolução de problemas que surgem no dia a dia de trabalho.

A realização de programas de educação continuada com o intuito de promover o desenvolvimento do pessoal de enfermagem, bem como a transmissão de informações e treinamento relativos as inovações tecnológicas constituem as atividades educativas desenvolvidas pelo enfermeiro.

Em relação as atividades de pesquisa, salientamos que já participamos da coleta de dados de trabalhos desenvolvidos por anestesistas do serviço; entretanto o presente estudo consiste na nossa primeira experiência específica na enfermagem.

O pessoal de enfermagem (auxiliares e técnicos de enfermagem) realiza as seguintes atividades:

- preparo do material estéril e de consumo, equipamentos e instrumental cirúrgico que serão utilizados nas cirurgias; - montagem das salas de cirurgias;

- atendimento das solicitações emanadas do anestesista e equipe cirúrgica (circulante de sala);

- recepção do paciente na sala de cirúrgia;

- atendimento das solicitações do paciente durante o ato anestésico - cirúrgico;

- transporte do paciente para a sala de recuperação pósanestésica;

- monitorização dos sinais vitais;

- observação e cuidados necessários com o curativo do local cirúrgico, soros e controle de diurese; - participação nos programas de educação continuada.

Para finalizar, um primeiro aspecto que salientamos foi a dificuldade na formação de uma equipe de enfermagem. As pessoas que vieram trabalhar no serviço não tinham conhecimento e experiência em relação a área de enfermagem cirúrgica. Assim, realizamos diversos programas de educação continuada na tentativa de oferecer crescimento pessoal e profissional. O trabalho foi árduo, entretanto, hoje a equipe de enfermagem é composta por doze pessoas, as quais possuem objetivos e metas comuns voltados para o aprendizado diário.

Outro aspecto foi a resistência de alguns médicos frente ao serviço de cirurgia ambulatorial, por falta de conhecimento e credibilidade. Esses ainda acreditam que o melhor para o paciente é ficar internado. Neste período foi realizado um trabalho de conscientização, para modificarmos conceitos e postura destes profissionais que aos poucos foram adaptando-se ao novo esquema.

O nosso próximo desafio consiste na implementação de um programa para preparar o paciente e familiares que serão submetidos a experiência da cirurgia ambulatorial, tendo como referência o estudo desenvolvido por ELLERTON ${ }^{7}$. A autora aborda um programa de preparo do paciente e familiares, no qual na semana anterior ao procedimento anestésico - cirúrgico, os mesmos comparecem ao hospital para assistirem uma exposição em grupo com slides sobre os procedimentos realizados e posteriormente visitam as dependências do centro cirúrgico. No final várias questões são levantadas e respondidas pelos enfermeiros que na oportunidade realizam as orientações pré-operatórias verbalmente e por escrito. A autora acrescenta que o nível de satisfação com o referido programa é elevado e principalmente auxilia a família a assumir os cuidados pós-operatórios do paciente com segurança.

Atualmente, o serviço realiza todos os procedimentos possíveis em cirurgia ambulatorial, com cinco salas cirúrgicas; temos em média mensalmente um número de quinhentos e cinqüenta procedimentos. A enfermagem recebe elogios dos médicos, administradores e clientes. Isso nos estimula a continuar aperfeiçoando cada vez mais o "Hospital Dia" e prestando um atendimento qualificado. Hoje atendemos as seguintes especialidades: geral; cabeça e pescoço; dermatologia; ginecologia; oftalmologia; ortopedia; otorrinolaringologia; pediatria; plástica; proctologia; urologia; tórax e vascular. Essas especialidades contam quando necessário com a presença do anestesiologista que desenvolve as seguintes técnicas: loco-regional, peridural, raqui, geral e local. A anestesia local consiste no maior número em torno de $60 \%$ e o restante dividido entre as outras técnicas, destacando os bloqueios peridural seguido de anestesia geral. 


\section{CONSIDERAÇÕES FINAIS}

Após a implementação de um serviço de cirurgia ambulatorial, concluímos ser gratificante o trabalho do enfermeiro nesta área, onde o sucesso deste serviço depende muito deste profissional e da equipe de enfermagem. Entendemos que a cirurgia ambulatorial tomará vulto em nosso meio e o enfermeiro tem que conquistar este espaço.

Acreditamos que nos próximos anos teremos uma reviravolta, onde a maioria das cirurgias será realizada ao nível ambulatorial, como já acontece em países como os EUA, no qual a criação de serviços ambulatoriais nos últimos vinte anos cresceu de forma progressiva e as tendências apontam para a continuação deste movimento, conforme atestam os trabalhos de $\mathrm{CRUZ}^{5}$; LLEWELLEYN ${ }^{9}$.

No âmbito da enfermagem brasileira, esta realidade significa um avanço e com certeza existe a necessidade de profissionais qualificados. Necessitamos desenvolver estudos a este respeito para obtermos respaldo num futuro breve. Ao nosso ver, podemos pensar nesta modalidade como uma provável especialidade, o enfermeiro visualiza o paciente como um todo, pois o cuidado é integral, ou seja, ele é acompanhado no pré, trans, pós-operatório sempre pela mesma equipe de enfermagem. Alcançando assim, conforme citado por PRAÇA \& AZEVEDO ${ }^{13}$ em um estudo, um nível de satisfação do paciente com a enfermagem em torno de $95 \%$ como excelente e os outros $5 \%$ como bom.

\section{ESTABLISHMENT OF AN AMBULATORY SURGERY SERVICE: THE ROLE OF NURSING IN THIS SCENERY}

The purpose of the present study is to present the trajectory of a nurse in establishing a service of ambulatory surgeries in a general hospital. It approaches the importance of such a service in the contemporary context, considering the activities developed by the nurse and nursing staff, as well as the challenges and conquests found in daily work.

KEY WORDS: ambulatory surgery, nurse activities, nursing staff

\section{IMPLANTACIÓN DE UN SERVICIO DE CIRUGÍA DE AMBULATORIO: EL PAPEL DE LA ENFERMERÍA EN ESE ESCENARIO}

El presente estudio tiene como objetivo presentar la trayectoria del enfermero en la impantación de un servicio de cirurgía de ambulatorio en un hospital general. Las autoras abordan la importancia de este servicio en el contexto actual, discurriendo sobre las actividades desarrolladas por el enfermero y equipo de enfermería, bien como los desafios y conquistas enfrentados en el dia a dia de trabajo.

TÉRMINOS CLAVES: cirugía de ambulatorio, actividades del enfermero, equipo de enfermería

\section{REFERÊNCIAS BIBLIOGRÁFICAS}

01. CÁRNIO, A.M. et al. Orientação pré-operatória a pacientes com catarata e indicação de cirurgia ambulatorial Rev.Bras. Enfermagem, Brasília, v. 48, n.1, p. 39-45, jan./mar. 1995.

02. CASTRO, A.V.G.A. et al. Assistência de enfermagem no pós-operatório imediato de pacientes submetidos a colecistectomia por minilapatomia em sistema ambulatorial - Relato de experiência. In: CONGRESSO BRASILEIRO DE ENFERMAGEM EM CENTRO CIRÚRGICO, 2., São Paulo, 1995. Anais. São Paulo: Sociedade Brasileira de Enfermeiros de Centro Cirúrgico, 1995. p. 108-110.
03. COLOGNA, M.H.Y.T. et al. Proposta de orientação no pré-operatório de cirurgias ambulatoriais ortopédicas. In: CONGRESSO BRASILEIRO DE ENFERMAGEM EM CENTRO CIRÚRGICO, 2. , São Paulo, 1995. Anais. São Paulo: Sociedade Brasileira de Enfermeiros de Centro Cirúrgico, 1995. p. 15-23.

04. CONSELHO FEDERAL DE MEDICINA - CFM regulamenta cirurgia ambulatorial. Resolução CFM n. 1409/94. Boletim de Urologia, v. 9, n. 4, p. 14, 1994.

05. CRUZ, L.D. Ambulatory surgery: the next decade. AORN J., v. 51, n. 1, p. 241-247, January, 1990.

06. DAVIS, E.D. Grande cirurgia ambulatorial. Trad. André L. de S. Melgaço, Giuseppe Taranto, Jacob I. Lemos. Rio de Janeiro: Interlivros, 1987. 
07. ELLERTON, M.L. Preparing kids and parents for surgery. Can. Nurse, v. 90 , n. 10, p. 25-27, November, 1994.

08. GUIDO, L.A. Construindo um marco conceitual a partir da teoria do alcance dos objetivos de Imogene King, para fundamentar a assistência de enfermagem ao cliente cirúrgico ambulatorial. In: CONGRESSO BRASILEIRO DE ENFERMAGEM EM CENTRO CIRÚRGICO, 2, São Paulo, 1995. Anais. São Paulo: Sociedade Brasileira de Enfermeiros de Centro Cirúrgico, 1995. p. 24 - 28.

09. LLEWELLYN, J.G. Short stay surgery: present practices, future trends. AORN J., v. 53, n. 5, p. 1179-1191, may 1991.

10. MATHIAS, J.M. Ambulatory surgery meeting stresses quality of care. AORN J., v. 45, n. 5, p. 1191-1200, may 1987.

11. NOCITE, J.R. Anestesia geral e condutiva em cirurgia ambulatorial. Rev. Bras. Anestesiol., v. 45, n. 1, p. 7-14, janeiro/fevereiro 1995.

12. OLIVEIRA FILHO, A.L. Anestesia para pacientes de curta permanência hospitalar. Rev. Bras. Anestesiol., n.33, p. 183 - 197, 1983.
13. PRAÇA, M.T.; AZEVEDO, F.L. Relato de experiência sobre atuação de enfermeira na unidade de cirurgia ambulatorial. In: CONGRESSO BRASILEIRO DE ENFERMAGEM EM CENTRO CIRÚRGICO, 2, São Paulo, 1995. Anais. São Paulo: Sociedade Brasileira de Enfermeiros de Centro Cirúrgico, 1995. p. 131-137.

14. ROZA, J.B. O papel do enfermeiro de centro cirúrgico. Rev. Gaúch. Enfermagem, Porto Alegre, v. 10, n. 2, p. 20-25, julho 1989.

15. SILVA, M.D'A.A. et al. Enfermagem na unidade de centro cirúrgico. 2. ed. São Paulo: Pedagógica e Universitária, 1997. p. 161-166: cirurgia ambulatorial.

16. WATSON, D.S.; SANGERMANO, C.A. Cirurgia ambulatorial. In: MEEKER, M.H.; ROTHROCK, J.C. Alexander/Cuidados de enfermagem ao paciente cirúrgico. 10. ed. Rio de Janeiro: Guanabara Koogan, 1997. Cap. 27, p. 1059-1077.

17. YAMASHITA, A.M. Anestesia ambulatorial. In: ORTENZI, A.V.; TARDELLI, M.A. Anestesiol. São Paulo: Atheneu, 1996. Cap. 39, p. 631-639. 